\title{
Elevations in EEG Power Spectra During the Recall of Sport Failure Amongst University Athletes
}

\author{
Laura A. Ceccarelli, Ryan J. Giuliano \\ Department of Psychology, University of Manitoba, Winnipeg, MB
}

\begin{abstract}
Previously, we showed that university athletes demonstrate cardiac reactivity resembling an acute stress response while recalling a previous sport failure. Athletes who reported higher levels of selfcompassion showed greater elevation of parasympathetic nervous system reactivity during recall of failure, and also showed more adaptive behavioural reactions, less maladaptive thoughts, and less negative affect during the task. Here, we analyzed changes in power spectra of the electroencephalogram (EEG) before, during, and after the recall of a previous sports failure, and whether individual differences in self-compassion or related constructs impact EEG changes during recall. Significant reactivity to, and recovery from, the recall task was observed across all EEG bandwidths: delta power decreased, and theta, low alpha, high alpha, low beta, and high beta power all increased from baseline to recall. Analogous EEG power changes were observed during recovery: delta power increased, while theta, low alpha, high alpha, low beta, and high beta power all decreased to baseline levels. Large gender differences were observed, with females generally showing greater EEG power across bandwidths during all phases of the experiment. Higher levels of self-compassion were associated with reduced theta power at baseline and during the recovery phase. Reactivity and recovery scores of EEG power were associated with self-reported self-esteem in the low alpha band: individuals reporting higher self-esteem showed greater increases in low alpha power during the recall task and greater decreases in low alpha power during recovery. These results are amongst the first to examine EEG power changes during experiences of acute stress and may have implications for improving how athletes recover from failures in sporting events.
\end{abstract}

Keywords: electroencephalogram, EEG, EEG power spectra, sports failure, self-compassion, selfesteem, athletes 


\section{Introduction}

The brain plays a central role in responding and adapting to stress (McEwen \& Gianaros, 2010). Yet, in contrast to the degree to which we understand the endocrine and cardiac response to acute stress and the neural contributions to stress reactivity in animal models (Ulrich-Lai et al., 2009), we know relatively little about how activity of the human brain is modulated during experiences of acute stress. What we do know about the effects of acute stressors on brain activity is largely based on studies utilizing social exclusion paradigms which elicit mild distress (e.g., Cyberball), as well as findings showing modulations of task-specific indices of brain function from pre- to post-exposure to an acute stressor. Given the notable heterogeneity across methods and results, more work is needed to isolate the influence of psychological stress on concurrent neural activity.

Studies of social exclusion in children and adults consistently report increases in frontal midline theta power to social exclusion or rejection events (Morales, Vollorani, \& Perez-Edgar, 2019; Tang et al., 2019; van Noordt et al., 2015). Findings from research using the Cyberball task, a paradigm shown to induce mild distress through participants being excluded from a virtual ball tossing game (Williams \& Jarvis, 2006), demonstrate increases in theta power at frontal midline electrodes on trials when participants are excluded from their turn to catch and toss the ball, relative to identical trials when it is not the participants turn but not because their turn was rejected (Cristofori et al., 2013). Other work shows that rejection events in Cyberball also elicit increases in power in the alpha and beta bandwidths, but it is only the increases in frontal midline theta power which tracks individual differences in participant distress (van Noordt et al., 2015). These findings converge with results from alternative paradigms inducing social rejection, which have demonstrated increases in frontal midline theta power time-locked to peer rejection events (Kortink et al., 2018; Morales et al., 2019; van der Molen et al., 2017).

These findings echo modulations in frontal midline theta that have been reported during cognitive tasks designed to induce stress; however, it should be noted that these studies find reductions in frontal midline theta power associated with increased stress (Gärtner et al., 2014; 2015). Similarly, a study of shooting performance under conditions of threat observed reductions in theta power from sources in the prefrontal cortex relative to control non-threatening blocks (Schlink et al., 2017). Such modulations of theta may not be restricted to frontal regions of the scalp, as recent research utilizing magnetoencephalography (MEG) suggests that theta oscillations in the temporal and parietal cortices are also impacted by acute stress (Quaedflieg et al., 2020).

Studies utilizing event-related potentials (ERPs) have reported changes in ERP components associated with exposure to acute stress. While modulations have been consistently reported at early stages of processing, the directionality of the impact of stress on the amplitude of different ERP components is mixed. Utilizing speeded arithmetic as a stressor, Qi and Gao have demonstrated an increase in N2 amplitudes and decrease in P3 amplitudes during stress relative to control blocks (Qi, Gao, \& Liu, 2018). In one study, the authors report amplitude increases across ERP components, seen as a phasic increase in amplitudes of the N1-N2-P3 components time-locked to targets during the Eriksen flanker task (Qi \& Gao, 2020). In another, effects on ERPs recorded after stress reductions in P2 and N2 amplitudes relative to a control group (Qi, Gao, \& Liu, 2017). Such heterogeneity has been reported by other groups, such as Dierolf and colleagues who have reported that acute stress enhanced amplitudes of the N2 and P3 in one study (Dierolf et al., 2018) and increased N2 amplitude but decreased P3 amplitude in another study (Dierolf et al., 2017). This variability in results is likely due to the fact that ERP components are heavily-influenced by the qualities of the stimulus events they are time-locked to (Luck, 2012), For these reasons, we propose that spectral measures of EEG are likely better indicators for isolating the stress-specific modulations of brain activity.

Interest in the brain's response to stress has grown among those working with elite athletes. Neurofeedback techniques have been developed using EEG, which involve monitoring EEG activity of individuals and giving verbal or visual feedback to these individuals designed to increase or decrease 
particular aspects of the EEG signal, with the goal of increasing attentional focus, relaxation, memory, and/or imagery (Shaw et al., 2012). Theoretically, athletes can utilize this immediate feedback to achieve a cortical state ideal for performance that can be replicated under performance conditions and improve self-regulation (Singer, 2002). Such training is typically achieved under neutral conditions to ensure an athlete is capable of reaching this optimal physiological state, and thus, most research using neurofeedback in sport has examined athletes' neural activity under neutral conditions. Common approaches to neurofeedback training among athletes has involved reducing theta $(4-7 \mathrm{~Hz})$ activity and increasing alpha (8-15 Hz) waves (Xiang et al., 2018). Specific attention has been paid to Sensorimotor Rhythm (SMR) power (12-15 Hz), thought to represent inhibition of somatosensory and sensorimotor processing (Kober et al., 2015; Sterman,1996), which may facilitate automatic processing involved in motor execution with which have been associated with superior sporting performance (Xiang et al., 2018).

Given the lack of research to date on the effects of acute stress on neural function in adults generally, and specifically, competitive athletes, we were interested in whether changes in electroencephalogram (EEG) power spectra could be observed during and after an acute laboratory psychosocial stressor. EEG power spectra in adults are typically examined through canonical frequency bands consisting of delta $(0.5-3 \mathrm{~Hz})$, theta $(3-7 \mathrm{~Hz})$, alpha $(7-12 \mathrm{~Hz})$, high alpha or SMR $(12-15 \mathrm{~Hz})$, beta $(15-20 \mathrm{~Hz})$, and high beta $(20-40 \mathrm{~Hz} \mathrm{~Hz})$ (Xiang et al., 2018). Previous work has established general cognitive correlates associated with activity in the separate EEG bandwidths, with delta power associated with general alertness (e.g., Sachdev et al., 2015), theta power related to selective attention and cognitive control (e.g., Buzzell et al., 2020; Orekhova et al., 2006), alpha power related to focused attention (e.g., Aftanas \& Golocheikine, 2001) and beta power related to active processing when ontask (e.g., Marek et al., 2018). Thus, changes in power spectra of EEG bandwidths as a function of acute stress might be informative as to the nature of systemic changes in cognition under stress.

Given the lack of previous research on the effects of acute psychosocial stress on EEG power in adults, we explored pre- to post-stress changes in EEG power across each of the predefined bandwidths of EEG. The primary purpose of the present study was to examine changes in EEG power across baseline, stress, and recovery phases and attempt to characterize EEG responding across these conditions among competitive athletes.

\section{Design and Participants}

\section{Methods}

Participants were university and national level athletes in Central Canada. A total of 80 athletes completed this laboratory-based, observational study. Participants were eligible to participate if they were currently selected to compete in their sport at a university or national level, free from psychological or physical conditions or medications that may alter their stress response, did not have a current head injury, and able to recall a recent sport failure or setback. Eligibility screening was completed online. Participants who completed the study and were included in the analyses were 80 adult athletes ( $M$ age $=21$; $S D=3.42$; range: 18 to 40 ) who were primarily single $(95 \%)$, Caucasian $(76.3 \%)$, university students $(96 \%)$, with slightly more participation from females $(56 \%)$ and represented a variety of sports with the most participation from track and field athletes (21.3\%).

\section{Measures}

Demographics. Participants reported their age, gender, marital status, current sport, year in sport at a university or national level and university major.

Self-compassion. Self-compassion was assessed using the 26-item Self-Compassion Scale (Neff, 2003b). Participants responded on a 5-point Likert scale ranging from 1 (almost never) to 5 (almost always). Six subscales assess the three facets of self-compassion and their opposing facets: mindfulness (over-identification), self-kindness (self-criticism) and common humanity (isolation). Negatively worded items were reverse scored. Means of each subscale were created and combined to 
create a grand self-compassion mean (Neff, 2003). Higher scores on this scale indicate higher levels of self-compassion. The Self-Compassion Scale has good test-retest reliability, discriminant and concurrent validity and good internal consistency reliability $(\alpha=.92)$ and scale items have been found to be reliable among athletic samples $(\alpha=.87$; Mosewich et al., 2011) including the present sample $(\alpha$ $=.91)$.

Self-esteem. The 10-item Rosenberg Self-Esteem Scale (RSES; Rosenberg, 1965) assessed self-esteem. Participants indicated the extent to which they agreed with each statement on a scale from 1 (strongly disagree) to 4 (strongly agree). Negatively worded items were reverse scored, and scores from all 10 items were summed. Higher scores represent higher levels of self-esteem. The scale shows good predictive, concurrent, construct validity and scale items were internally consistent in other $(\alpha=$ .87; Rosenberg, 1965) and in the present sample $(\alpha=.82)$. The scale shows acceptable psychometric properties when used with athletic samples (e.g., Mosewich et al., 2011) and has been used previously as a control variable alongside self-compassion (Mosewich et al., 2011).

Imagery ability. Given that imagery ability may impact participants' reactivity during the stress induction (Kwekkeboom, 2000), imagery ability was included as a possible control variable in this study. The Motivational General-Arousal (MG-A) subscale of the Motivational Imagery Ability Measure for Sport (MIAMS), was chosen in order to assess participant's ability to generate emotional experiences associated with sport (e.g., anxiety) using imagery (Gregg \& Hall, 2006). This subscale assesses participants ease of forming the image, and intensity of the emotional experience generated by the image. To complete this scale, participants were asked to generate images associated with four different sport scenarios (e.g., feeling anxious before a sporting competition), and rate the ease of forming the image (4-items) and the emotional experience (4-items) created by the image on scale from 1 (no emotion) to 7 (very strong emotion). Emotion and ease were assessed separately (Gregg \& Hall, 2006). The MG-A subscale of the MIAMS has shown acceptable reliability ( $\alpha=.74$ emotion; $\alpha=.73$ ease), including within the present sample ( $\alpha=.70$ emotion; $\alpha=.69$ ease), among athletic samples.

\section{Laboratory Measures}

EEG. Using a ProComp Infiniti (Thought Technology, Montreal, Canada) multi-modal biofeedback system, recordings were utilized from three, 120-second phases: Before (baseline assessment), during (reactivity) and following stress induction (recovery). A single-channel EEG electrode was used and recorded from Cz. Prior to recording, the skin on the participants' scalp and ears was prepared using NuPrep, and Ten20 conductive paste. EEG signals were recorded using Biograph Infiniti software. The sampling rate was 256 samples/second. Mastoid reference electrodes were placed on both of the participants' earlobes. Electrode skin contact was checked and all impedance levels were kept under $10 \mathrm{k} \Omega$. This software contains a function for auto-rejection of artifact signals, which was set to $>/=50 \mathrm{uV}$.

Image quality. Athletes rated the extent to which the generated failure image was easy to generate, arousing, clear, meaningful, emotional, and useful (6-items) on a scale from 1 (not at all easy to form) to 7 (very easy to form). A mean score was computed from the sum of six items to assess overall image quality. This manipulation check ensured the effectiveness of the stress induction. This measure was developed based on recommendations from imagery researchers (see Gregg \& Hall, 2006; Hammond et al., 2012; Lang, 1979).

\section{Procedures}

Recruitment, eligibility and baseline assessment. Upon attaining institutional ethics approval, we recruited participants from two Canadian universities and a national sport centre, through requests to teams, posters, and word of mouth. We emailed interested participants the online eligibility survey. Eligible participants provided informed consent and completed the baseline survey online which included measures of self-compassion, self-esteem, and demographics.

Laboratory session. Two days prior to a scheduled laboratory session, participants were briefed regarding pre-laboratory session eligibility criteria. Participants were asked to ensure that they were free from substances (e.g., alcohol, drugs, or medications) or physical conditions (e.g., concussion, lack of/poor sleep or illness) that may impact their body's physical responses to stress 
(Laborde et al., 2017; Svendsen et al., 2016), in order to ensure that their physiological data was as reliable as possible. Participants were asked to reschedule their session if they did not meet the prelaboratory session criteria. The first author conducted sessions at laboratories at the universities and the sports centre. After a brief orientation to study procedures, the researcher connected the participant to the equipment used to record EEG responses (ProComp Infiniti, Thought Technology, Montreal, Canada).

The researcher then instructed the participant to remain calm and relaxed for a two-minute acclimation period, followed by a two-minute baseline assessment of EEG. A two-minute acclimation period is considered acceptable in order to alleviate participants' anxieties or nerves and to help them to feel comfortable with being connected to the physiological recording equipment (Heathers et al., 2014; Laborde et al., 2017). The instructions for the acclimation and baseline assessments were to sit comfortably and relaxed with both of their feet flat on the floor, hands on their thighs and palms facing up (Laborde et al., 2017). Participants were told to remain as still as possible, as movement may interfere with the recording. Next, participants underwent a stress induction. To induce a stress response, participants were asked to imagine a recent sport failure or setback with their eyes closed, for two minutes, using a guided imagery script read aloud by the researcher. Participants were instructed to remain seated with their eyes closed and provided the same instructions that were delivered during the baseline recording (feet flat on the floor, hands on their thighs and palms facing up) and were again instructed to remain as still as possible. The imagery script was read aloud to prompt participants to image their past failure for the entire two-minute period. Prompts were provided followed by brief pauses to allow participants to generate their images. The imagery script provided prompts to promote elaboration of participant's images and the emotion associated with their failure experience. Further, providing ongoing prompts helped to ensure that participants were thinking about the failure scenario for the duration of the stress induction. The imagery script was developed based on imagery best practice (Hammond et al., 2012; Lang, 1979) and by consulting with a sports imagery expert. The imagery script was pilot tested prior to commencing data collection to ensure it extended for the duration of the two-minute time frame. Moreover, the same researcher (the first author) read and followed the rehearsed script for all participants to provide uniform delivery. The imagery script for the stress induction was as follows:

Remember a time when you failed... Maybe you made a costly mistake, failed to meet an important goal, or experienced a setback in your sport progress...... Imagine this experience....... In your mind, really try to take yourself back to this experience...... Remember your expectations leading up to this... Remember the pressures that you felt... Imagine what you were looking forward to and your hopes... Then remember the situation unfolding as it did... Remember where you were, what your surroundings looked like, who was there.............Take yourself back to the stressful situation in as much detail as possible. Really focus on the feelings that you had..... Disappointment, anger, frustration, despair... Try to remember those feelings in as much detail as possible...... Really allow yourself to feel them... Remember the changes in your body......tension, anxiousness, uneasiness....... Imagine this scenario in as much detail as possible...... Even after this moment or situation had passed, notice any feelings that remain: tension, regret, uneasiness........ Really try to take yourself back to the feelings and emotions that you experienced......... Now, please take a deep breath and gently open your eyes.

The stress induction was followed by a two-minute recovery period where participants were instructed to relax their body and their mind with their eyes open. The researcher then disconnected the participant from the equipment and the participant completed the psychological laboratory measures (see laboratory measures in methods). Participants were debriefed and provided with an explanation of the study's purpose and then thanked for their involvement. 


\section{Descriptive Statistics}

\section{Results}

Zero-order correlations between all variables of interest with EEG Power during each phase of the experiment are shown below (Table 2). Means and standard deviations of EEG power during baseline, stressor, and recovery are reported in the Supplementary materials (Supplemental Table 1).

Age was not associated with any outcomes of interest. However, gender was significantly correlated with EEG power across bandwidths and time points. To follow up on associations with gender, independent samples $t$-tests were performed to compare values for males and values on EEG power measures taken during the baseline, stressor, and recovery phases (Table 3). At baseline, females showed greater EEG power in the delta $[t(78)=-4.28, p<.001]$, theta $[t(78)=-3.45, p=.001]$, high alpha $[t(78)=-2.39, p=.019]$, low beta $[t(78)=-3.02, p=.003]$, and high beta bandwidths $[t(78)=$ -2.33, $p=.022]$. During the stressor, females showed greater EEG power in the delta $[t(77)=-3.82, p<$ $.001]$, low beta $[t(77)=-2.40, p=.019]$, and high beta bandwidths $[t(77)=-2.38, p=.020]$. During recovery, females showed greater EEG power in the delta $[t(78)=-3.77, p<.001]$, theta $[t(78)=-2.80$, $p=.006]$, high alpha $[t(78)=-2.03, p=.046]$, low beta $[t(78)=-2.66, p=.010]$, and high beta bandwidths $[t(78)=-2.03, p=.046]$.

Table 1. Zero-order Correlations Between Self-report Variables and EEG Power ( $\mu$ V) during Baseline, Stressor, and Recovery Measurements.

\begin{tabular}{|c|c|c|c|c|}
\hline & Age & Gender & $\begin{array}{l}\text { Self- } \\
\text { Esteem }\end{array}$ & $\begin{array}{l}\text { Self- } \\
\text { Compassion }\end{array}$ \\
\hline Age & - & -.104 & -.009 & .103 \\
\hline Gender & - & - & .103 & -.083 \\
\hline Self-esteem & - & - & - & $-.599^{\star \star \star}$ \\
\hline Self-compassion & - & - & - & - \\
\hline Baseline Delta & -.118 & $.436^{\star \star \star *}$ & $.245^{\star}$ & -.181 \\
\hline Baseline Theta & -.112 & $.364^{\star \star}$ & .155 & $-.240^{*}$ \\
\hline Baseline Low Alpha & -.097 & .170 & .125 & -.116 \\
\hline Baseline High Alpha & -.130 & $.261^{*}$ & .112 & -.184 \\
\hline Baseline Low Beta & -.031 & $.324^{\star \star}$ & .130 & $-.225^{\star}$ \\
\hline Baseline High Beta & -.022 & $.255^{*}$ & .085 & $-.209^{t}$ \\
\hline Stressor Delta & -.115 & $.400^{* \star *}$ & .093 & -.155 \\
\hline Stressor Theta & -.126 & .141 & .153 & $-.199^{t}$ \\
\hline Stressor Low Alpha & -.124 & .013 & $.266^{*}$ & -.131 \\
\hline Stressor High Alpha & -.063 & .178 & .090 & -.118 \\
\hline Stressor Low Beta & -.043 & $.263^{*}$ & .156 & $-.217^{t}$ \\
\hline Stressor High Beta & -.031 & $.262^{*}$ & .168 & $-.214^{t}$ \\
\hline Recovery Delta & -.160 & $.393^{\star * \star}$ & .128 & -.151 \\
\hline Recovery Theta & -.083 & $.302^{\star *}$ & .121 & $-.250^{\star}$ \\
\hline Recovery Low Alpha & -.052 & .131 & .153 & -.126 \\
\hline Recovery High Alpha & -.117 & $.224^{*}$ & .123 & -.161 \\
\hline Recovery Low Beta & -.019 & $.288^{*}$ & .105 & $-.212^{t}$ \\
\hline Recovery High Beta & .008 & $.224^{*}$ & .080 & -.175 \\
\hline
\end{tabular}

${ }^{t}, p<.1 ;{ }^{*}, p<.05 ;{ }^{* \star}, p<.01 ;{ }^{* * *}, p<.001$
Table 2. Independent Samples t-tests of Gender Differences in EEG Power $(\mu \mathrm{V})$ during the Baseline, Stressor, and Recovery Measurements.

\begin{tabular}{|c|c|c|c|c|}
\hline Bandwidth & Location & Males & Females & $p$ \\
\hline \multirow[t]{5}{*}{ Baseline } & $\begin{array}{l}\text { Delta } \\
\text { Theta }\end{array}$ & $\begin{array}{l}10.86 \\
9.97\end{array}$ & $\begin{array}{l}12.78 \\
11.72\end{array}$ & $\begin{array}{c}<.001^{\star \star *} \\
.001^{\star *}\end{array}$ \\
\hline & Low Alpha & 7.16 & 8.23 & .133 \\
\hline & High Alpha & 5.98 & 7.41 & $.019^{*}$ \\
\hline & Low Beta & 4.92 & 5.90 & $.003^{* *}$ \\
\hline & High Beta & 6.30 & 7.40 & $.022^{\star}$ \\
\hline \multirow[t]{6}{*}{ Stressor } & Delta & 9.95 & 11.53 & $<.001^{\star * *}$ \\
\hline & Theta & 11.11 & 12.06 & .216 \\
\hline & Low Alpha & 10.38 & 10.51 & .909 \\
\hline & High Alpha & 8.37 & 9.96 & .116 \\
\hline & Low Beta & 5.43 & 6.28 & $.019^{*}$ \\
\hline & High Beta & 6.71 & 7.70 & $.020^{*}$ \\
\hline \multirow[t]{6}{*}{ Recovery } & Delta & 10.82 & 12.58 & $<.001^{\star \star *}$ \\
\hline & Theta & 9.87 & 11.28 & $.006^{\star *}$ \\
\hline & Low Alpha & 6.90 & 7.67 & .246 \\
\hline & High Alpha & 5.78 & 6.98 & $.046^{*}$ \\
\hline & Low Beta & 4.75 & 5.59 & $.010^{*}$ \\
\hline & High Beta & 6.23 & 7.07 & $.046^{*}$ \\
\hline
\end{tabular}

NOTE: all Baseline and Recovery pairwise comparisons tested at $t(78)$; Stressor pairwise comparisons tested at $t(77)$. ${ }^{*}, p<.05 ;{ }^{* *}, p<.01 ;{ }^{* * *}, p<.001$

In terms of self-reported participant characteristics, self-compassion and self-esteem showed associations with EEG power. Self-compassion was negatively associated with theta power during baseline $[r(78)=-.240, p=.032]$ and recovery measurements $[r(78)=-.250, p=.025]$, and was also negatively associated with baseline low beta power $[r(78)=-.225, p=.044]$. Similar trends were observed between self-compassion scores and: baseline high beta power; theta, low beta, and high beta power during the stressor; and low beta during recovery. Self-esteem was positively associated with delta power during the baseline $[r(78)=.245, p=.028]$ and low alpha power during the stressor $[r(77)=.266, p=.018]$. 


\section{Analysis of EEG Power by Bandwidth and Time}

We performed a $3 \times 6$ repeated measures ANOVA with factors of Time (baseline, stressor, recovery) $\times$ Bandwidth (delta, theta, low alpha, high alpha, low beta, high beta) on EEG power values illustrated in Figure 1 below. The repeated measures ANOVA revealed significant main effects of Time $\left[F(2,156)=53.914, p<.001 ; \boldsymbol{\eta}_{\mathrm{p}}^{2}=\right.$ $.409]$ and Bandwidth $[F(5,390)=$ $\left.139.268, p<.001 ; \boldsymbol{\eta}_{\mathrm{p}}{ }^{2}=.641\right]$.

Collapsing across bandwidth, EEG power was highest during the stressor $(M=8.297, S E=.263 ; 95 \%$ Confidence Interval $=7.876$ to 8.719 ) and lowest during the recovery period $(M=8.092, S E=.195 ; 95 \%$ Confidence Interval $=7.641$ to 8.417 ) . Collapsing across time, EEG power generally decreased from lower frequency (e.g., delta and theta) to higher frequency (e.g., alpha and beta) bandwidths. However, these main effects were qualified by a significant Time $\times$ Bandwidth interaction $[F(10$, $\left.780)=50.055, p<.001 ; \boldsymbol{\eta}_{\mathrm{p}}{ }^{2}=.391\right]$, such that each bandwidth showed significant changes in EEG power from baseline to stressor, as well as from

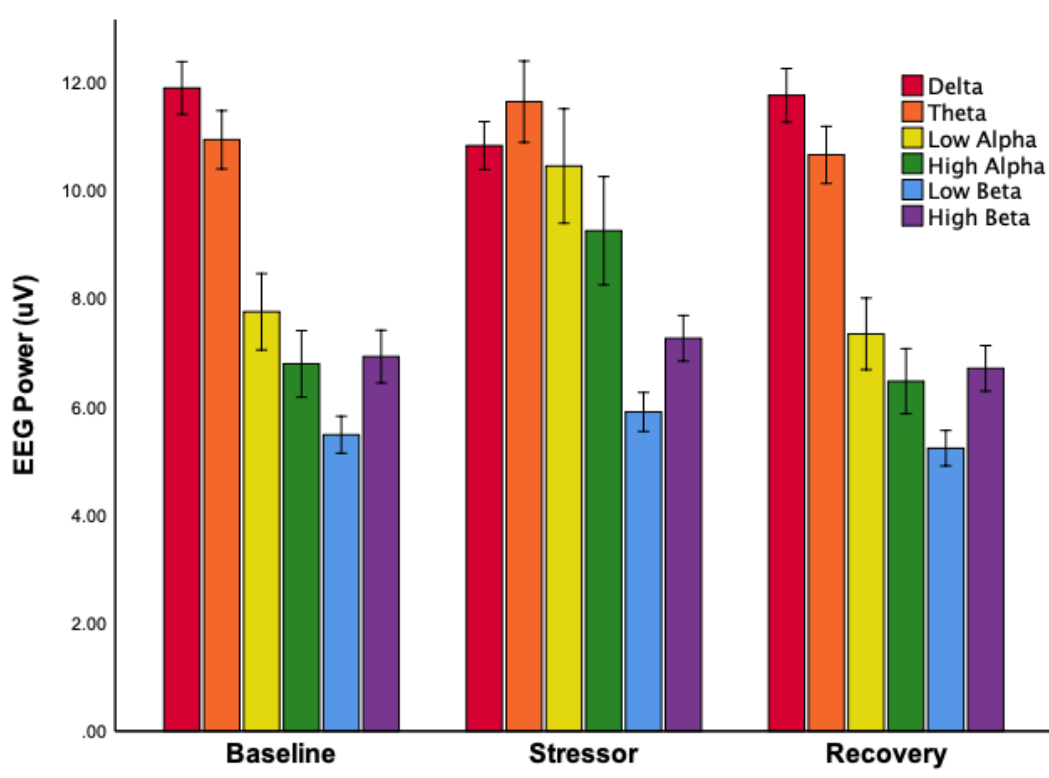

Figure 1. Bar plot of EEG power during Baseline, Stressor, and Recovery. Error bars shown are $95 \%$ confidence intervals. stressor to recovery.

To illustrate the change in EEG power from baseline to stressor to recovery, difference scores for stress reactivity (stressor minus baseline) and stress recovery (recovery minus stressor) are shown in Figure 2 below. Delta power was reduced during the stressor relative to baseline, but all other bandwidths showed increases in EEG power during the stressor relative to baseline. After the stressor, all bandwidths showed significant changes in EEG power during the recovery period, with values trending back towards baseline values.
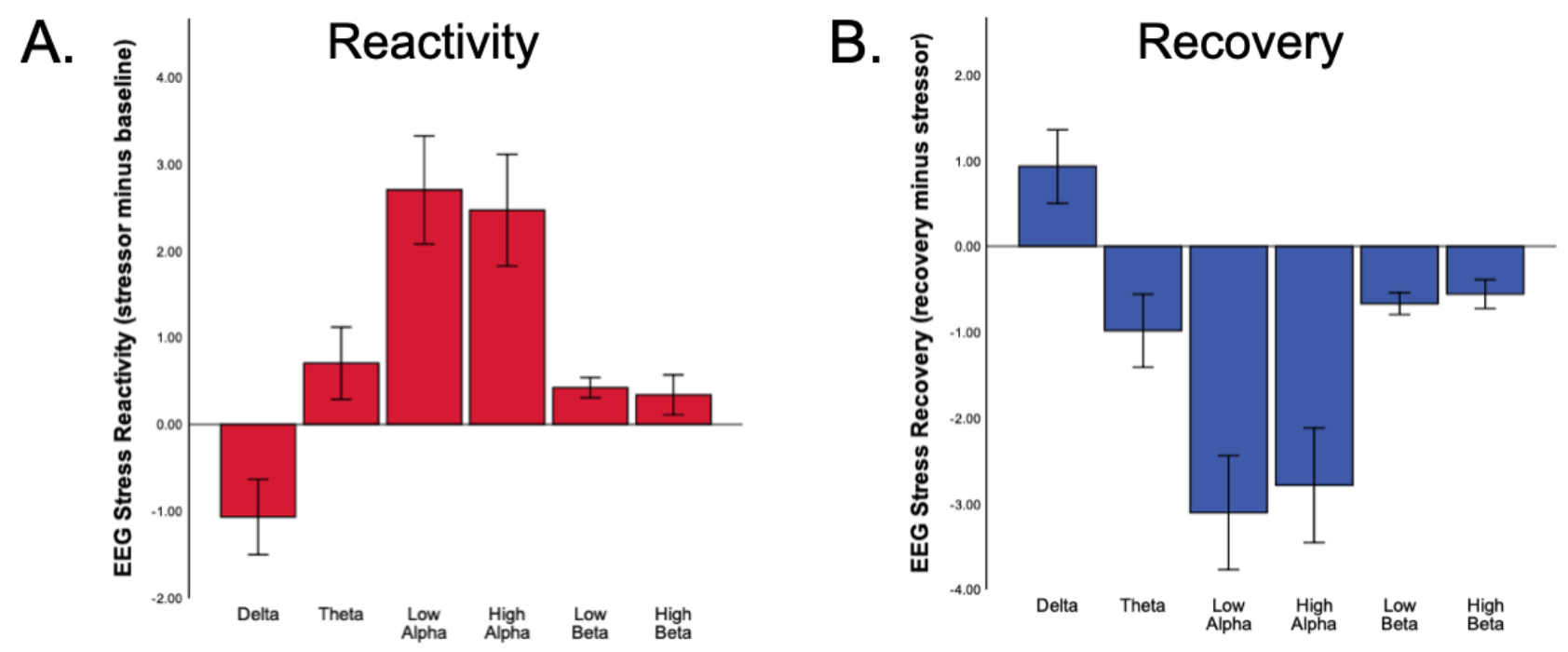

Figure 2. Bar plots of relative EEG power change during A) stress reactivity and B) stress recovery. Error bars shown are $95 \%$ confidence intervals. 
Following up on associations reported above for gender, self-esteem, and self-compassion with EEG power during the stress and recovery periods, we examined correlations with difference scores of the magnitude of stress reactivity and stress recovery. No significant correlations were observed between age, gender, or self-compassion with EEG power change to the stressor or EEG power change to the recovery period. However, self-esteem was significantly associated with EEG power stress reactivity $[r(77)=.310, p=.005]$ and recovery $[r(77)=-.269, p=.017]$. As shown in Figure 3 below, individuals reporting higher self esteem showed greater increases in the low alpha power during the stressor relative to baseline, and larger reductions in low alpha power during the recovery relative to the stressor.
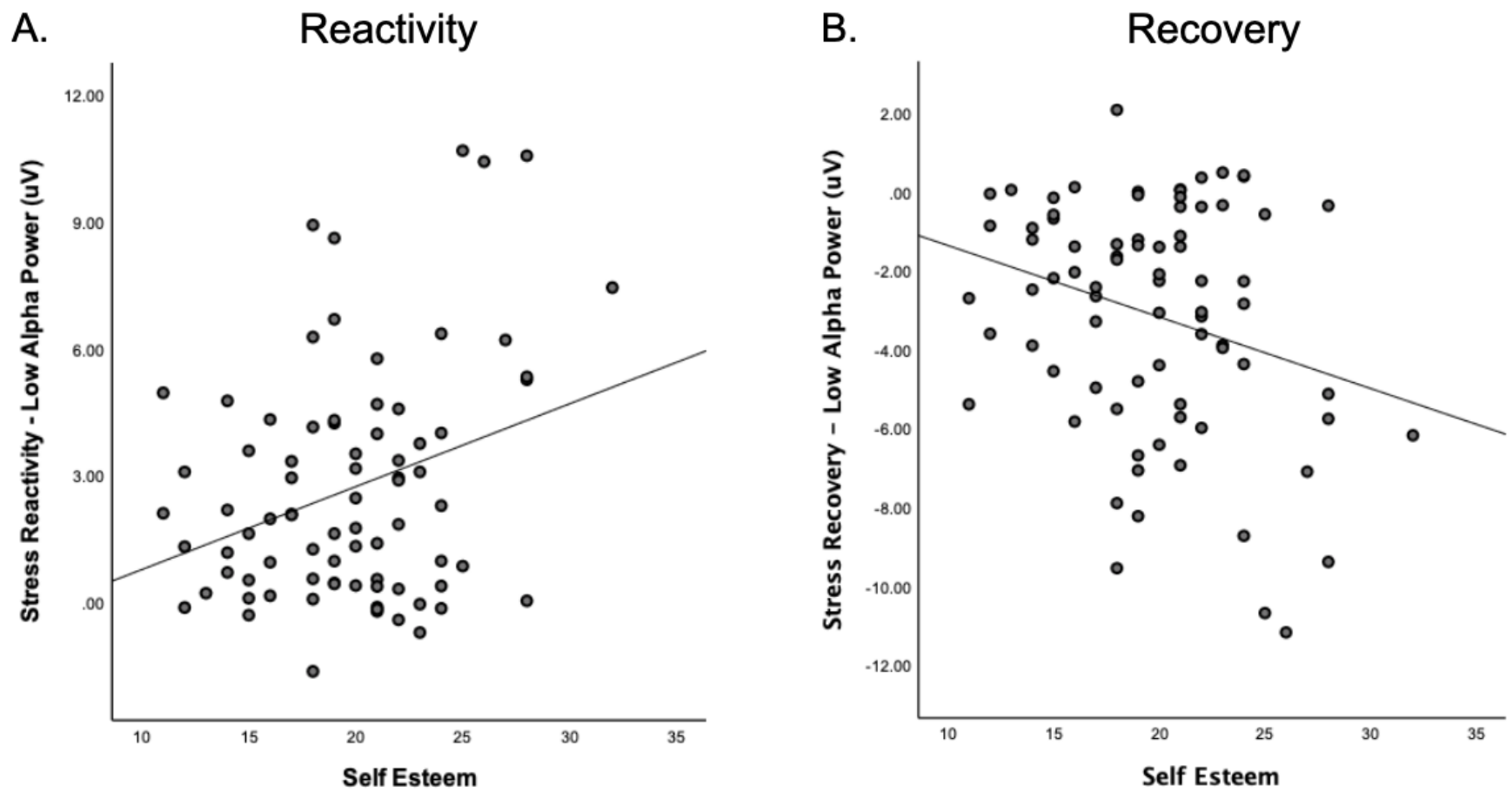

Figure 3. Scatterplots of association between self-reported Self Esteem and Low Alpha Power during A) stress reactivity and B) stress recovery.

\section{Discussion}

This study examined EEG power before, during, and after a task during which athletes were asked to imagine a previous sport failure. We observed broad bandwidth changes in EEG power during recall and a return to baseline EEG power across all bandwidths during a recovery phase. Gender differences were also observed in broad bandwidth EEG power, seen as greater power in female than male athletes during all phases of the experiment. Individual differences in self-compassion and selfesteem showed some evidence of associations with EEG power during each phase of the task, all of which we unpack below.

Our results support previous research positing that stress, including psychological stress, impacts neural activity. Specifically, several studies utilizing forms of social exclusion as a stress induction technique have reported increased EEG power in the theta frequency band, which may reflect up-regulation of neural activity underlying expectation violation (Cristoferi et al., 2013; Morales et al., 2019; Tang et al., 2019; van Noordt et al., 2015). Other studies have reported modulation of the alpha frequency band, reflecting changes in arousal levels irrespective of visual input (e.g., eyes open or closed; Barry et al., 2007). One study utilizing a shooting task under threat of experimenter fire observed modulations of EEG power in both the theta and alpha bands, seen as decreases in power after participants pulled the trigger (Schlink et al., 2017). Changes in other bandwidths across 
conditions reported here may be attributable to ocular changes (e.g., eyes open vs. eyes closed), and should be further examined in future research (Barry et al., 2007). However, given the lack of a control group in the present study, it is not clear which EEG bandwidths showed modulations in power across the experiment that would not have been observed in participants who did not undergoing the imagery procedure.

Given the increasing popularity of neurofeedback training with athletes (Xiang et al., 2018), our results suggest that further research understanding the brain under stressful as opposed to neutral conditions may provide additional training targets. Our findings suggest that EEG power is modulated by stress relative to rest, so perhaps neurofeedback training for athletes should consider conducting training or assessment under simulated levels of arousal. Results do not provide much specificity in bandwidths modulated by an imagined failure task, so future research should utilize control groups. For instance, it would be beneficial to include a neutral or positive (e.g., positive imagery) control to compare the cortical responses of these conditions to those of the stressful condition and further tease apart the impact of stress on these processes. Further, including a control group of non-athletes would allow for the opportunity to generalize our results to a broad, adult sample. This may also afford the opportunity to determine if sport involvement offers any unique benefits in terms of the brain's responses to stress.

We found that across all phases of the experiment, females showed greater power across EEG bandwidths compared to males. This finding is consistent with other research that has demonstrated that females showed higher resting EEG power compared to males (Jausovec \& Jausovec, 2010; Wada et al., 1994; Wada et al., 1994). In one study, women showed higher EEG power compared to males during baseline and photo stimulation conditions (Wada et al., 1994). This suggests that gender differences may persist across task demanding conditions. However, future research should continue to explore the impact of gender on the brain's response to stress, given that a number of studies have reported gender differences in the response to acute stress (e.g., Hamidovic et al., 2020).

Our results should be interpreted in light of several limitations. First, our sample included only elite athletes, which limits the generalizability of our findings to adults broadly. Second, the lack of a control group limits our ability to conclude that the imaging of a past failure elicited a stress response over and above what a control group may have reported over the course of this lab visit, absent of the imagery task. However, the changes in cardiac physiology we have previously reported in this sample are consistent with what is observed in a canonical stress response (Ceccarelli et al., 2019).

Taken together, these findings offer preliminary evidence that brain activity, as reflected by EEG power, is modulated in athletes during an imagined sport failure task. Future research is needed to refine what bandwidths of EEG might be selectively influenced by imagining a sport failure over and above what is seen in non-athletes and over above what is seen in less stressful versions of the imagery task. Such results could have implications for neurofeedback training in elite athletes and provide bandwidths that could be targeted for improving athletes' performance under moments of pressure when risk of failure is high, as well as potentially improving athletes' ability to recover after moments of failure to minimize carryover of the failure to future performance. 


\section{References}

Aftanas, L. I., \& Golocheikine, S. A. (2001). Human anterior and frontal midline theta and lower alpha reflect emotionally positive state and internalized attention: High-resolution EEG investigation of meditation. Neuroscience Letters, 310(1), 57-60.

Barry, R., Clarke, A., Johnstone, S., Magee, C., \& Rushby, J. (2007). EEG differences between eyesclosed and eyes-open resting conditions. Clinical Neurophysiology, 118, 2765-2773.

Buzzell, G. A., Troller-Renfree, S. V., Wade, M., Debnath, R., Morales, S., Bowers, M. E., Zeanah, C. H., Nelson, C. A., \& Fox, N. A. (2020). Adolescent cognitive control and mediofrontal theta oscillations are disrupted by neglect: Associations with transdiagnostic risk for psychopathology

Ceccarelli, L., Giuliano, R. J., Glazebrook, C., \& Strachan, S. (2019). Self-compassion and psychophysiological recovery from recalled sport failure. Frontiers in psychology, 10, 1564.

Cristofori, I., Moretti, L., Harquel, S., Posada, A., Deiana, G., Isnard, J., Mauguiere, G., \& Sirigu, A. (2013). Theta signal as the neural signature of social exclusion. Cerebral Cortex, 23(10), 24372447.

Dierolf, A. M., Fechtner, J., Böhnke, R., Wolf, O. T., \& Naumann, E. (2017). Influence of acute stress on response inhibition in healthy men: An ERP study. Psychophysiology, 54(5), 684-695.

Dierolf, A. M., Schoofs, D., Hessas, E. M., Falkenstein, M., Otto, T., Paul, M., ... \& Wolf, O. T. (2018). Good to be stressed? Improved response inhibition and error processing after acute stress in young and older men. Neuropsychologia, 119, 434-447.

Gärtner, M., Grimm, S., \& Bajbouj, M. (2015). Frontal midline theta oscillations during mental arithmetic: effects of stress. Frontiers in behavioral neuroscience, 9, 96.

Gärtner, M., Rohde-Liebenau, L., Grimm, S., \& Bajbouj, M. (2014). Working memory-related frontal theta activity is decreased under acute stress. Psychoneuroendocrinology, 43, 105-113.

Gregg, M. \& Hall, C. (2006). Measurement of motivational imagery abilities in sport. Journal of Sports Sciences, 24(9), 961-971.

Hamidovic, A., Van Hedger, K., Choi, S. H., Flowers, S., Wardle, M., \& Childs, E. (2020). Quantitative meta-analysis of heart rate variability finds reduced parasympathetic cardiac tone in women compared to men during laboratory-based social stress. Neuroscience \& Biobehavioral Reviews, 114, 194-200.

Hammond, T., Gregg, M., Hrycaiko, D., Mactavish, J., \& Leslie-Toogood, A. (2012). The effects of a motivational-general mastery imagery intervention on the imagery ability and sport confidence of inter-collegiate golfers. Journal of Imagery Research in Sport and Physical Activity, 7(1), 1-20.

Heathers, J., Fink, E., Kuhnert, R-L., \& de Rosnay, M. (2014). Blood volume pulse (BVP) derived vagal tone (VT) between 5 and 7 years of age: A methodological investigation of measurement and longitudinal stability. Developmental Psychobiology, 56(1), 23-35.

Jausovec, N. \& Jausovec, K. (2010). Resting brain activity: Differences between genders. Neuropsychologia, 48, 3918-3925.

Kober, S.E., Witte, M., Stangl, M., Väljamäe, A., Neuper, C., \& Wood, G. (2015). Shutting down sensorimotor interference unblocks the networks for stimulus processing: An SMR neurofeedback training study. Clinical Neurophysiology, 126 (1), pp. 82- 95.

Kortink, E. D., Weeda, W. D., Crowley, M. J., Moor, B. G., \& van der Molen, M. J. (2018). Community structure analysis of rejection sensitive personality profiles: A common neural response to social evaluative threat?. Cognitive, Affective, \& Behavioral Neuroscience, 18(3), 581-595.

Kwekkeboom, K. (2000). Measuring imagery ability: Psychometric testing of the Imaging Ability Questionnaire. Research in Nursing and Health, 23, 301-309.

Laborde, S., Mosley, E., \& Thayer, J. (2017). Heart rate variability and cardiac vagal tone in psychophysiological research- Recommendations for experiment planning, data analysis, and data reporting. Frontiers in Psychology, 8(213), 1-18.

Lang, P. (1979). A bio-informational theory of emotional imagery. Psychophysiology, 16(6), 495-512.

Luck, S. J. (2012). Event-related potentials. In H. Cooper, P. M. Camic, D. L. Long, A. T. Panter, D. Rindskopf, \& K. J. Sher (Eds.), APA handbooks in psychology®. APA handbook of research 
methods in psychology, Vol. 1. Foundations, planning, measures, and psychometrics (p. 523546). American Psychological Association.

McEwen, B. S., \& Gianaros, P. J. (2010). Central role of the brain in stress and adaptation: Links to socioeconomic status, health, and disease. In Annals of the New York Academy of Sciences (Vol. 1186, pp. 190-222). Blackwell Publishing Inc.

Morales, S., Vallorani, A., \& Pérez-Edgar, K. (2019). Young children's behavioral and neural responses to peer feedback relate to internalizing problems. Developmental cognitive neuroscience, 36 , 100610.

Mosewich, A., Kowalski, K., Sabiston, C., Sedgwick, W., \& Tracy, J. (2011). Self-Compassion: A potential resource for young women athletes. Journal of Sport and Exercise Psychology, 33, 103-123.

Neff, K. (2003b). The development and validation of a scale to measure self-compassion. Self and Identity,2, 223-250.

Orekhova, E. V., Stroganova, T. A., Posikera, I. N., \& Elam, M. (2006). EEG theta rhythm in infants and preschool children. Clinical Neurophysiology, 117(5), 1047-1062.

Qi, M., \& Gao, H. (2020). Acute psychological stress promotes general alertness and attentional control processes: An ERP study. Psychophysiology, 57(4), e13521.

Qi, M., Gao, H., \& Liu, G. (2017). Effect of acute psychological stress on response inhibition: an eventrelated potential study. Behavioural brain research, 323, 32-37.

Qi, M., Gao, H., \& Liu, G. (2018). The effect of mild acute psychological stress on attention processing: an ERP study. Experimental brain research, 236(7), 2061-2071.

Quaedflieg, C. W. E. M., Schneider, T. R., Daume, J., Engel, A. K., \& Schwabe, L. (2020). Stress impairs intentional memory control through altered theta oscillations in lateral parietal cortex. Journal of Neuroscience, 40(40), 7739-7748.

Rosenberg, M. (1965). Society and the adolescent self-image. Princeton, NJ: Princeton University Press.

Sachdev, R. N. S., Gaspard, N., Gerrard, J. L., Hirsch, L. J., Spencer, D. D., \& Zaveri, H. P. (2015). Delta rhythm in wakefulness: Evidence from intracranial recordings in human beings. Journal of Neurophysiology, 114(2), 1248-1254. https://doi.org/10.1152/jn.00249.2015

Schlink, B. R., Peterson, S. M., Hairston, W. D., König, P., Kerick, S. E., \& Ferris, D. P. (2017). Independent component analysis and source localization on mobile EEG data can identify increased levels of acute stress. Frontiers in Human Neuroscience, 11, 310.

Shaw, L., Zaichkowsky, L., \& Wilson, V. (2012). Setting the balance: Using biofeedback and neurofeedback with gymnasts. Journal of Clinical Sport Psychology, 6, 47-66.

Singer, R.N. (2002). Preperformance state, routines, and automaticity: What does it take to realize expertise in self-paced events? Journal of Sport \& Exercise Psychology, 24, 359-375.

Sterman, M. (1996). Physiological origins and functional correlates of EEG rhythmic activities: Implications for self-regulation. Biofeedback \& Self Regulation, 21 (1), pp. 3-33.

Tang, A., Lahat, A., Crowley, M. J., Wu, J., \& Schmidt, L. A. (2019). Neurodevelopmental differences to social exclusion: An event-related neural oscillation study of children, adolescents, and adults. Emotion, 19(3), 520.

Ulrich-Lai, Y. M., \& Herman, J. P. (2009). Neural regulation of endocrine and autonomic stress responses. Nature reviews neuroscience, 10(6), 397-409.

van der Molen, M. J. W., Dekkers, L. M., Westenberg, P. M., van der Veen, F. M., \& van der Molen, M. W. (2017). Why don't you like me? Midfrontal theta power in response to unexpected peer rejection feedback. Neurolmage, 146, 474-483.

van Noordt, S. J., White, L. O., Wu, J., Mayes, L. C., \& Crowley, M. J. (2015). Social exclusion modulates event-related frontal theta and tracks ostracism distress in children. Neurolmage, 118, 248-255.

Wada, Y., Takizawa, Y., Zheng-Yan, J., \& Yamaguchi, N. (1994). Gender differences in quantitative EEG at rest and during photic stimulation in normal young adults. Clinical

Electroencephalography, 25(2), 81-85. 
Williams, K. D., \& Jarvis, B. (2006). Cyberball: A program for use in research on interpersonal ostracism and acceptance. Behavior research methods, 38(1), 174-180.

Xiang, M. Q., Hou, X. H., Liao, B. G., Liao, J. W., \& Hu, M. (2018). The effect of neurofeedback training for sport performance in athletes: A meta-analysis. Psychology of Sport and Exercise, 36, 114-122. 


\section{Supplementary Materials}

Table 1. Means and Standard Deviations of EEG Power $(\mu \mathrm{V})$ during Baseline, Stressor, and Recovery Measurements.

\begin{tabular}{llll} 
Bandwidth & Baseline & Stressor & Recovery \\
\hline Delta & $11.89(2.18)$ & $10.83(1.98)$ & $11.76(2.21)$ \\
Theta & $10.94(2.41)$ & $11.64(3.36)$ & $10.66(2.35)$ \\
Low Alpha & $7.75(3.15)$ & $10.45(4.72)$ & $7.35(2.95)$ \\
High Alpha & $6.79(2.74)$ & $9.26(4.46)$ & $6.47(2.69)$ \\
Low Beta & $5.48(1.52)$ & $5.90(1.61)$ & $5.23(1.47)$ \\
High Beta & $6.93(2.17)$ & $7.27(1.89)$ & $6.71(1.88)$
\end{tabular}

$N=79$. 